\title{
Forma Simplificada de Determinação das Funções de Atraso Filanovsky-Matkhanov e Propostas de Modificações
}

\author{
Douglas David Baptista de Souza e Sidnei Noceti Filho
}

\begin{abstract}
Resumo: Neste trabalho, é mostrada uma forma simplificada de determinação de funções de atraso Filanovsky-Matkhanov. Tais funções, quando comparadas com outras funções clássicas como, por exemplo, Bessel, apresentam tanto um atraso maior quanto uma maior seletividade. São também discutidas modificações que preservam a ordem da função original, seja alargando a banda passante sem alterar o tempo de atraso ou aumentando o atraso sem modificar a banda. São mostrados resultados obtidos por simulações.
\end{abstract}

Palavras-chave-Aproximação de Bessel, funções de atrasos.

Abstract: In this work, a simplified way of determining the Filanovsky-Matkhanov's delay functions is shown. These functions, when compared with other classical ones, such as Bessel, for instance, present a larger delay as well as selectivity. Several types of modifications are discussed, preserving the order of the original function, for instance, increasing the bandwidth without changing the delay time or the delay without changing the band. Results from simulations are showed.

Keywords-Bessel approximation, delay functions.

\section{INTRODUÇÃO}

Redes de atraso têm importantes aplicações em sistemas de processamento de sinais, particularmente em redes de alta velocidade e em sistemas de controle. Um sistema idealizado (não fisicamente realizável), que atrasa um sinal sem distorção (ver Fig. 1), apresenta uma função de transferência $T(s)$ dada por (1), a qual apresenta uma largura de banda infinita e um tempo de atraso $T_{0}$ constante para todas as freqüências, resultando na saída em um sinal sem distorção.

$$
T(s)=K e^{-T_{0} S} \text {. }
$$

Conseqüentemente, a resposta em freqüência $T(\omega)$ é:

$$
T(\omega)=K e^{-j \omega T_{0}}=Y(\omega) / X(\omega)
$$

onde $Y(\omega)$ e $X(\omega)$ são as transformadas de Fourier dos sinais de saída $y(t)$ e de entrada $x(t)$, respectivamente.

Então, $|T(\omega)|=K$ e $\theta(\omega)=-\omega T_{0} \quad(K>0)$ (equação de uma reta com inclinação $-T_{0}$ que passa pela origem). A transformada de Laplace inversa, dada por (3), mostra que o

Douglas David Baptista de Souza e Sidnei Noceti Filho, LINSE Laboratório de Circuitos e Processamento de Sinais, Depto. de Eng. Elétrica, Universidade Federal de Santa Catarina, Florianópolis, SC, E-mails: \{douglas, sidnei\}@linse.ufsc.br. sinal de saída do sistema idealizado é simplesmente multiplicado por uma constante $K$ e sofre um atraso no tempo igual a $T_{0}$.
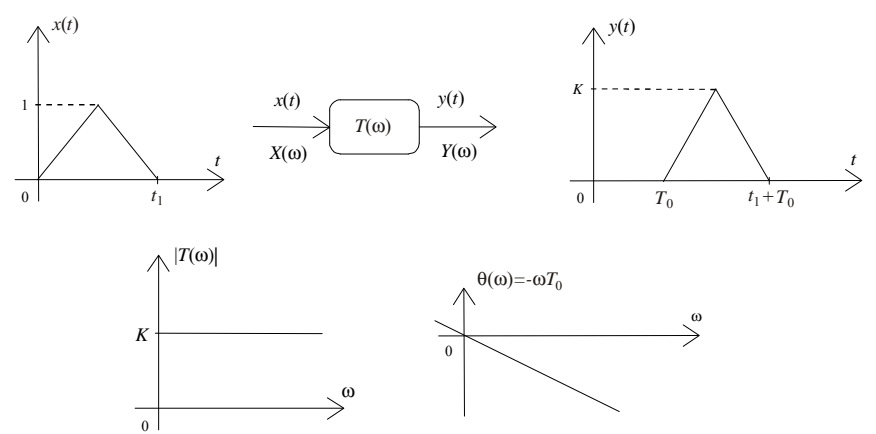

Fig. 1. Transmissão sem distorção $(K>0)$.

$$
y(t)=K x\left(t-T_{0}\right)
$$

$\mathrm{Na}$ prática, soluções aproximadas são obtidas com funções reais racionais em $s$ na forma $T(s)=N(s) / D(s)$. No entanto, não se pode afirmar $a$ priori qual a melhor solução sob o ponto de vista da banda desejada e do atraso requerido. Por exemplo, um sinal com conteúdo espectral limitado pode exigir do filtro uma banda limitada para maximizar a relação sinal/ruído. Assim, o projetista deve ter opções de funções de aproximação que apresentam diferentes larguras de banda e diferentes atrasos.

Para uma dada função, sempre é possível alterar o valor do atraso através de escalamento de freqüência por um fator $a$. No entanto, existe uma relação biunívoca entre a banda e o valor do atraso devido à propriedade escalar da transformada de Fourier. Se $f(t) \leftrightarrow F(\omega)$, então $f(a t) \leftrightarrow(1 / a) F(\omega / a)$.

Então, para uma determinada banda, diferentes atrasos podem ser obtidos com a utilização de diferentes funções, as quais têm sido propostas na literatura por diferentes autores [1]-[3].

Uma das funções mais usadas por projetistas é a função de aproximação de Bessel (BS) [4], que é uma aproximação de ordem $n$ da função em (1). Assim, quanto maior for a ordem $n$, melhor será a aproximação da equação e, portanto, maior será a faixa de freqüência em que o atraso permanecerá constante, mas também com o filtro ficando mais seletivo. Devido às limitações inerentes da função $\mathrm{BS}$, os projetistas necessitam de várias opções para a obtenção de diferentes bandas e de diferentes atrasos. 
Uma destas funções é a Filanovsky-Matkhanov (FM) [2], cuja forma simplificada de determinação é mostrada neste trabalho. Também são sugeridas modificações na função original FM, com o intuito de se obter diferentes atrasos para uma mesma banda ou obter diferentes bandas sem alterar a fase.

Este trabalho está organizado da seguinte forma. Na Seção II, é discutida a função de atraso FM e é apresentada uma expressão geral simplificada que apresenta menos parâmetros variáveis do que a expressão apresentada em [2]. Na Seção III, as funções FM são comparadas com as funções BS e são apresentados resultados de simulação para várias ordens. $\mathrm{Na}$ Seção IV, são discutidas funções FM Modificadas. $\mathrm{Na}$ Seção V, são apresentadas as conclusões deste trabalho.

\section{FunÇÕES DE AtRASO FILANOVSKY-MATKHANOV}

O processo para se obter as funções de atraso FilanovskyMatkhanov (FM) [2] pode ser dividido em alguns passos. Inicialmente, consideram-se as funções seno mostradas em (6) como uma aproximação no domínio do tempo da resposta ao impulso ideal. Em seguida, acha-se a transformada de Laplace dessa aproximação. A representação no domínio da freqüência será uma função não-realizável. Logo, é necessária a realização de outra aproximação para se obter uma FT do tipo polinomial.

$$
h(t)=\left\{\begin{array}{cc}
0 & 0<t<\tau \\
\operatorname{Ksen}^{2 m} \beta(t-\tau) & \tau<t<\tau+\frac{T}{2} . \\
0 & t>\tau+\frac{T}{2}
\end{array}\right.
$$

A partir de (6), adotando-se $m=1, K=4 / T$ e $\tau=T / 4$, onde $T=2 \pi / \beta$ é o período do seno e $\beta$ é um fator de escala genérico, obtêm-se as funções de atraso FM, apresentadas em (7).

$$
h(t)=\left\{\begin{array}{cc}
0 & 0<t<\tau \\
\frac{4}{T} \operatorname{sen}^{2} \beta(t-\tau) & \tau<t<\tau+\frac{T}{2} . \\
0 & t>\tau+\frac{T}{2}
\end{array}\right.
$$

A transformada de Laplace de (7) é

$$
F(s)=\frac{8 \beta^{2}\left(1-e^{-(s T / 2) s}\right) e^{-(\tau) s}}{T s\left(s^{2}+4 \beta^{2}\right)} .
$$

A expressão em (8) pode ser reescrita como

$$
F(s)=\frac{2}{\pi} \frac{\operatorname{senh}\left(\frac{\pi s}{2 \beta}\right) e^{-(\tau+T / 4) s}}{\left[(s / 2 \beta)^{2}+1\right](s / \beta)}
$$

Para transformar (9) em uma FT realizável, deve-se efetuar a seguinte aproximação:

$$
\operatorname{senh}\left(\frac{\pi s}{2 \beta}\right) \approx \frac{\pi s}{2 \beta}\left(1+\frac{s^{2}}{4 \beta^{2}}\right)
$$

Através de um simples algebrismo, substituindo-se (10) em (9), tem-se que

$$
F(s) \approx e^{-(\tau+t / 4) s}
$$

Como $\tau=T / 4$ e $T=2 \pi / \beta$, e tomando a normalização $s=(\beta / 2) s^{\prime \prime}$, pode-se reescrever (11) como

$$
F(s) \approx e^{-(\pi / 2) s^{\prime \prime}} .
$$

Fazendo-se $s^{\prime \prime}=j \omega$ e considerando que

$$
\operatorname{sen}\left(\frac{\pi \omega}{2}\right)=\frac{\pi \omega}{2} \prod_{i=1}^{\infty}\left(1-\frac{\omega^{2}}{4 i^{2}}\right)
$$

e

$$
\cos \left(\frac{\pi \omega}{2}\right)=\prod_{i=0}^{\infty}\left(1-\frac{\omega^{2}}{(2 i+1)^{2}}\right)
$$

tem-se

$$
F(j \omega) \approx \prod_{i=0}^{\infty}\left(1-\frac{\omega^{2}}{(2 i+1)^{2}}\right)-j \frac{\pi \omega}{2} \prod_{i=1}^{\infty}\left(1-\frac{\omega^{2}}{4 i^{2}}\right)
$$

gerando a FT mostrada em (16).

$$
T\left(s^{\prime \prime}\right)=\frac{A_{1}}{\prod_{i=0}^{n_{1}}\left(s^{\prime \prime 2}+(2 i+1)^{2}\right)+s^{\prime \prime} A_{2}\left(\prod_{i=1}^{n_{2}}\left(s^{\prime \prime 2}+4 i^{2}\right)\right)}
$$

onde

$$
\begin{gathered}
A_{1}=\prod_{i=1}^{n_{1}}(2 i+1)^{2} \\
A_{2}=\left(\frac{1,043 \prod_{i=1}^{n_{1}}(2 i+1)^{2}}{\prod_{i=1}^{n_{2}}\left(4 i^{2}-1\right)}\right) .
\end{gathered}
$$

Para se obter $T(s)$, basta substituir $s^{\prime \prime}=(2 / \beta) s$ em (16). Deste modo:

$$
T\left(\frac{2 s}{\beta}\right)=\frac{A_{1}}{\prod_{i=0}^{n_{1}}\left(\left(\frac{2 s}{\beta}\right)^{2}+(2 i+1)^{2}\right)+\left(\frac{2 s}{\beta}\right) A_{2}\left(\prod_{i=1}^{n_{2}}\left(\left(\frac{2 s}{\beta}\right)^{2}+4 i^{2}\right)\right)} .
$$


Em (20), é mostrada uma forma simplificada da função FM determinada em (19), no sentido em que não possui o parâmetro designado por $\beta$ (presente na expressão geral de [2]). Esse parâmetro, após alguns algebrismos, pode ser eliminado da equação geral porque nada mais é do que um parâmetro de normalização. Portanto, os parâmetros variáveis passam a ser apenas $n_{2}$ e $n_{1}$ presentes em (18).

$$
T\left(s^{\prime}\right)=\frac{\prod_{i=1}^{n_{1}}(2 i+1)^{2}}{\prod_{i=0}^{n_{1}}\left(s^{\prime 2}+(2 i+1)^{2}\right)+s^{\prime}\left(\frac{1,043 \prod_{i=1}^{n_{1}}(2 i+1)^{2}}{\prod_{i=1}^{n_{2}}\left(4 i^{2}-1\right)}\right)\left(\prod_{i=1}^{n_{2}}\left(s^{\prime 2}+4 i^{2}\right)\right)}
$$

É possível mostrar que a ordem $n$ das funções obtidas é dada por

$$
\begin{array}{cl}
\text { Caso par: } & n=2\left(n_{2}+1\right), \text { com } n_{2}=n_{1} \\
\text { Caso ímpar: } & n=2 n_{2}+1, \text { com } n_{2}=n_{1}+1
\end{array}
$$

Com a equação geral dada em (19), considerando ordens até $n=10$, considerando $\beta$ variando de 1 até 4,64 diferentes funções de transferência são obtidas, sendo que apenas 28 delas são estáveis. Como podemos prescindir do parâmetro $\beta$ (normalizações posteriores podem ser efetivadas) e as funções instáveis não nos interessam, as funções FM até a ordem 10 podem ser determinadas diretamente da Tabela I. Funções de qualquer ordem podem ser obtidas com a aplicação de (20).

Conforme apresentado em (6), sugere-se que uma resposta ao impulso ideal pode ser aproximada por outras funções seno de ordem par. Foram efetuadas aproximações para $m=2,3$, e 4, porém os resultados obtidos não foram satisfatórios, pelo aparecimento de ripple na resposta em magnitude, overshoot na resposta ao degrau e ondulações relativamente grandes no atraso de fase.

A mudança da ordem da função seno, que aproxima a resposta ao impulso, reflete-se na variação do fator $\mathrm{A}_{2}$, apresentado em (18). Pode-se observar em (19) que $\mathrm{A}_{2}$ será uma constante multiplicativa que aparece apenas nos coeficientes dos termos de ordem ímpar. Logo, variando-se o valor de $\mathrm{A}_{2}$ em uma determinada quantidade, obtém-se a mesma variação nos termos de ordem ímpar do denominador da FT.

$\mathrm{Na}$ função FM de décima ordem mostrada no final da Tabela I $\left(n_{2}=n_{1}=4\right)$, observou-se que melhores resultados foram obtidos ao diminuir o valor de $\mathrm{A}_{2}$ em uma faixa de até $10 \%$ do valor original.

A Tabela II apresenta a FT de décima ordem também em função de $A_{2}$. São mostradas também as funções obtidas quando esse termo decresce $5 \%$ e $10 \%$ em relação ao valor original.
TABELA I

CAsos Estáveis de FunÇões (FM) ATÉ A ORDEM 10

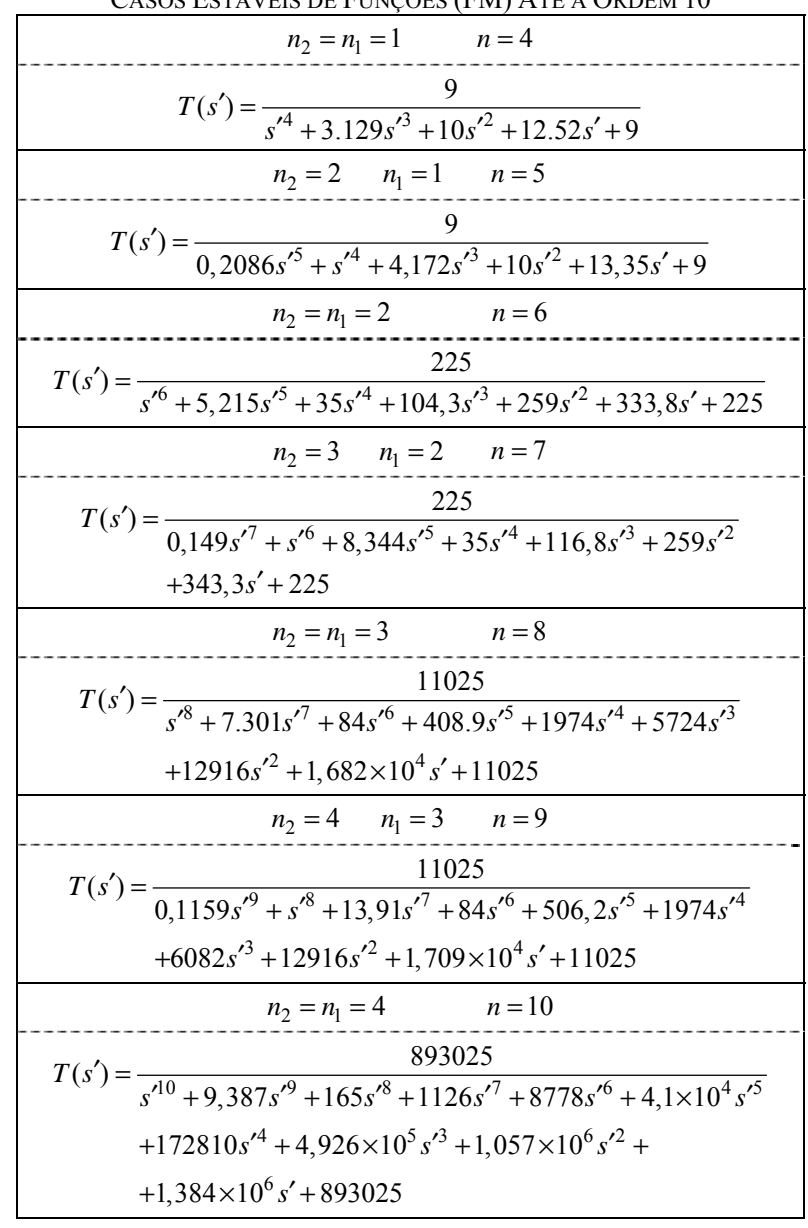

TABELA II

FUNÇÕES FM DE ORDEM 10 OBTIDAS COM A VARIAÇÃO DE $\mathrm{A}_{2}$

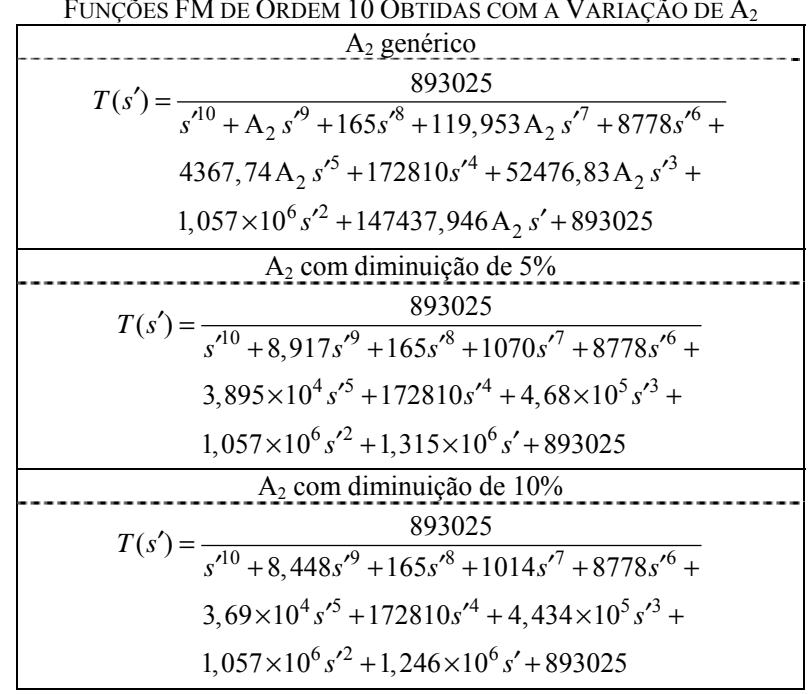

\section{COMPARAÇÃO ENTRE AS FunÇÕES FM E BS. RESUltados DE SimulaÇÃO}

As características das funções devem ser comparadas após a realização de algum tipo de normalização. Consideramos não ter nenhum sentido comparar funções como foram inicialmente concebidas, pois quando um determinado tipo de 
normalização é efetuado, os resultados podem ser completamente alterados devido à propriedade escalar da transformada de Fourier, já discutida neste trabalho. Os tipos de normalização mais freqüentemente utilizados são:

i) Funções de mesma ordem são normalizadas de modo a apresentar a mesma atenuação, por exemplo, $3 \mathrm{~dB}$, no limite da banda normalizada de $1 \mathrm{rad} / \mathrm{s}$.

Se a função obtida não apresenta naturalmente a desejada atenuação $A_{\max }$ em $1 \mathrm{rad} / \mathrm{s}$, na função original chamamos o argumento de $s^{\prime}$. Posteriormente, determina-se numericamente a freqüência $\omega_{N}$, através da função $\left|T\left(\omega^{\prime}\right)\right|_{\mathrm{dB}}=20 \log \left|T\left(s^{\prime}\right)\right|_{s^{\prime}=j \omega^{\prime}}$, na qual se tem a atenuação $A_{\max }$ desejada (ver Fig.2). Após, faz-se uma mudança de variável, substituindo-se $s^{\prime}$ por $s^{\prime}=\omega_{N} \bar{s}$ na função $T\left(s^{\prime}\right)$. Assim, no limite da banda passante normalizada, será obtida a desejada atenuação. Nesse caso, a freqüência dos pólos fica dividida por $\omega_{N}\left(\bar{s}_{k}=s_{k}^{\prime} / \omega_{N}\right)$. Então, os tempos de atraso ficarão multiplicados por $\omega_{N}$.

$$
\bar{\tau}_{g}(\omega)=\tau_{g}^{\prime}(\omega) \times \omega_{N}
$$

ii) As funções comparadas apresentam a mesma ordem e a mesma atenuação, por exemplo $40 \mathrm{~dB}$, no limite da banda de rejeição normalizada $\bar{\omega}_{S}=1 \mathrm{rad} / \mathrm{s}$. O método de determinação da função normalizada é análogo ao apresentado no item anterior.

iii) Para funções de mesma ordem, os atrasos de grupo são normalizados para apresentar o mesmo atraso de grupo na origem $\bar{\tau}_{g}(0)=1 \mathrm{~s}$, ou o mesmo valor do atraso de grupo médio em uma banda de interesse $\left(\bar{\tau}_{\text {gmedio }}=1 \mathrm{~s}\right)$. Seja uma função que apresenta na origem um atraso $\tau_{g}^{\prime}(0)=\tau_{x}$. Assim, o atraso normalizado $\bar{\tau}_{g}(0)$ será $\bar{\tau}_{g}(0)=\tau_{g}^{\prime}(0) / \tau_{x}=1 \mathrm{~s}$. Então, os novos pólos serão $\bar{s}_{k}=s_{k}^{\prime} \times \tau_{x}$. A normalização correspondente na função é $s^{\prime}=\bar{s} / \tau_{x}$.

Nos casos i) e ii), uma posterior desnormalização para uma freqüência $\omega_{a}$ qualquer pode ser obtida por uma simples mudança de variáveis $\bar{s}=s / \omega_{a}$.

No caso iii), uma posterior desnormalização para a obtenção de um $\tau_{g}(0)$ qualquer pode ser obtida por uma simples mudança de variáveis $\bar{s}=\tau_{g}(0) \times s$.

As Fig. 3 e 4 apresentam a magnitude, o atraso de fase e a resposta ao impulso das funções BS e FM normalizadas por $\omega_{N}$ (ver Tabela III), tal que essas apresentem uma atenuação de $3 \mathrm{~dB}$ na freqüência de $1 \mathrm{rad} / \mathrm{s}$. As ordens consideradas foram $n=4,6,8$ e 10 . As diferentes características que se destacam são: para uma mesma atenuação no limite da banda passante e para uma mesma ordem, as funções FM apresentam uma maior seletividade e um maior valor de atraso em uma faixa de freqüência maior. Isso ao custo do aparecimento de ripple nas funções de atraso, o que caracteriza uma maior não-linearidade da fase.

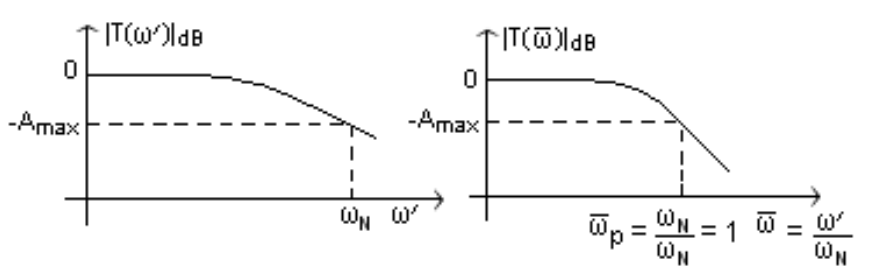

(a)

(b)

Fig. 2. (a) Função antes do escalamento; (b) função após o escalamento.

TABELA III

VALORES DE $\omega_{N}$ OBTIDOS NUMERICAMENTE PARA AS OITO FUNÇÕES ESTUDADAS

\begin{tabular}{|ccc|}
\hline & $\omega_{N}(\mathrm{BS})$ & $\omega_{N}(\mathrm{FM})$ \\
\hline$n=4$ & 2,114 & 1,65 \\
$n=6$ & 2,70 & 2,02 \\
$n=8$ & 3,18 & 2,32 \\
$n=10$ & 3,6 & 2,56 \\
\hline
\end{tabular}

\section{FUnÇÕES FM MODIFICADAS}

São discutidas, nesta seção, quatro formas de modificar as funções FM sem alteração da ordem da função original.

i) Aumento do Atraso para uma mesma banda:

Isto pode ser obtido com a variação de $\mathrm{A}_{2}$, discutida na seção II. O preço que se paga é uma deterioração da linearidade da fase. A Fig. 5 mostra um exemplo de uma função FM de ordem 10, com $\mathrm{A}_{2}$ decrescendo em $5 \%$ e $10 \%$.

ii) Aumento da Banda sem alteração da fase e, conseqüentemente, do atraso:

Isto é conseguido incluindo zeros em simetria especular na função FM. A Fig. 6 mostra um exemplo onde a banda foi aumentada com a simples colocação de um par de zeros reais em $\pm 3,3 \mathrm{rad} / \mathrm{s}$, gerando um numerador $N_{\mathrm{FM}}(s)=$ $-\left(s^{2}-10,89\right)$.

Respeitando a simetria especular, os zeros podem ser complexos ou reais. Nesse exemplo, a função FM possui ordem 8.

iii) Aumento da banda dobrando o valor do atraso:

Se $D_{\mathrm{FM}}(s)$ é o denominador de uma função FM, então ao incluir no numerador o polinômio $D_{\mathrm{FM}}(-s)$, a banda se tornará teoricamente infinita e o atraso obtido será o dobro do atraso da função original. A Fig.7 mostra um exemplo onde a função FM possui ordem 10.

iv) Aumento do atraso e da linearidade da fase sem alteração da banda:

Isto se obtém com a clássica equalização de fase obtida pela cascata do filtro original com uma seção all-pass.

\section{CONCLUSÕES}

Neste trabalho, foi apresentada uma forma simples de determinação das funções de atraso Filanovsky-Matkhanov que mostram características vantajosas em relação à função de Bessel. Foram também apresentadas formas de alterar as características temporais e/ou fase sem alterar as características de amplitude e vice-versa. As simulações foram efetuadas com o software SG2, disponível no endereço eletrônico http://www.linse.ufsc.br/ sidnei. 


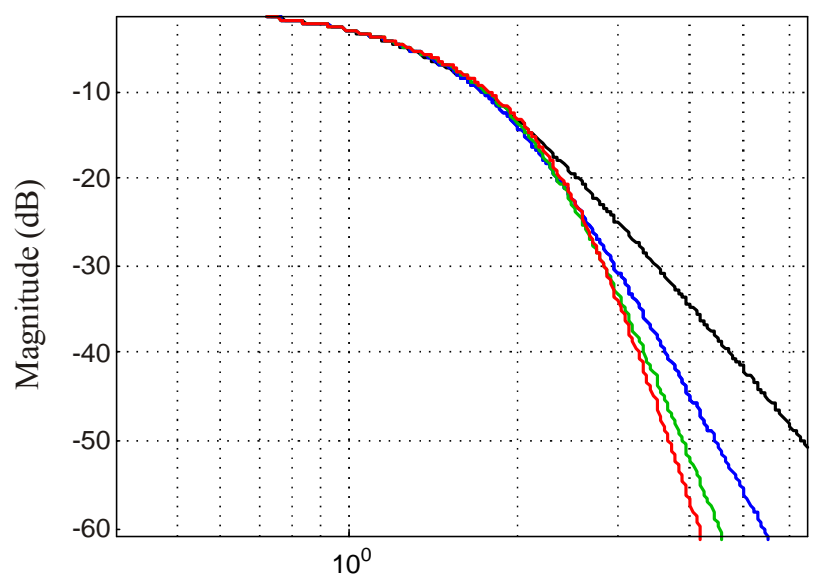

Freqüência (rad/s)

(a)

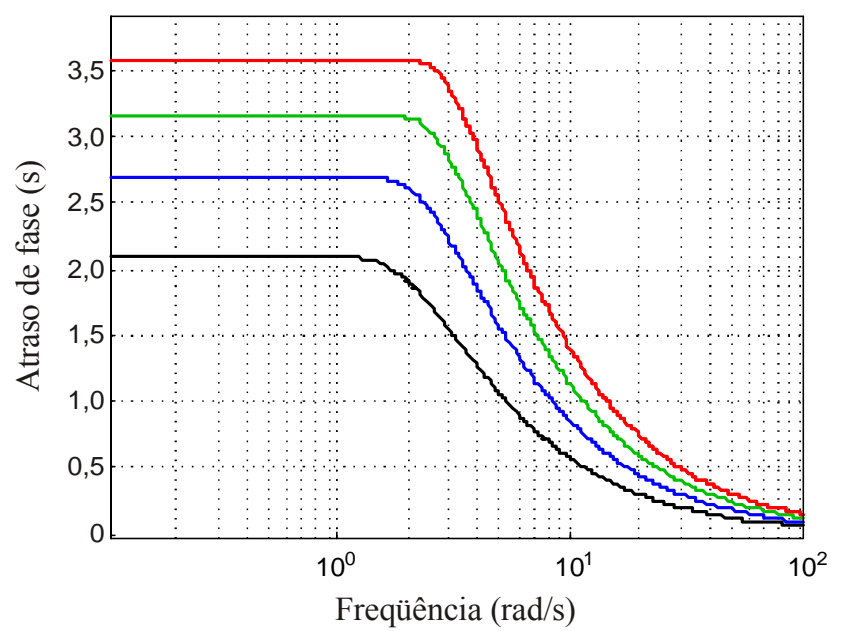

(b)

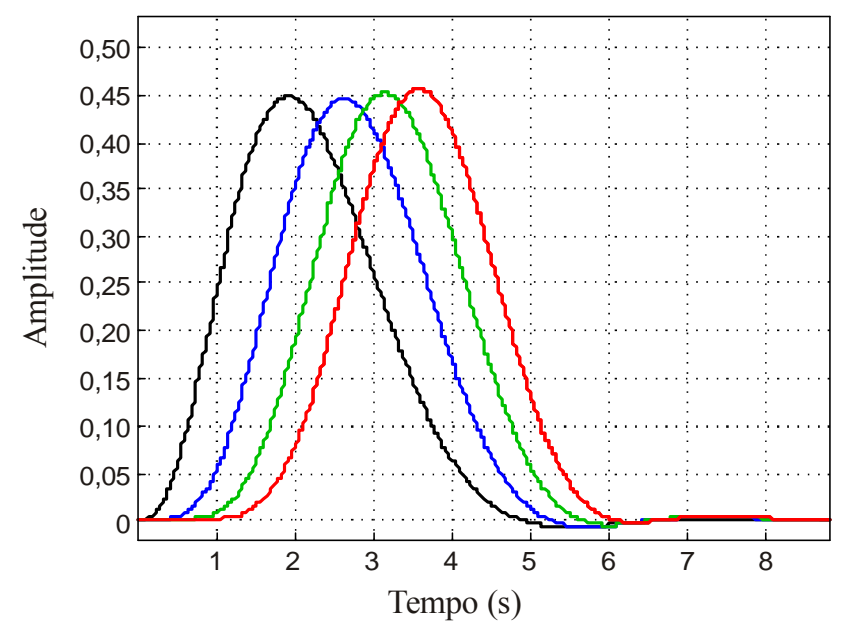

(c)

Fig. 3. Funções BS para $A_{\max }=3 \mathrm{~dB}$ em $1 \mathrm{rad} / \mathrm{s}$. (a) Magnitude; (b) atraso de fase; (c) resposta ao impulso. $n=4$ (preto), $n=6$ (azul), $n=8$ (verde), $n=10$ (vermelho).

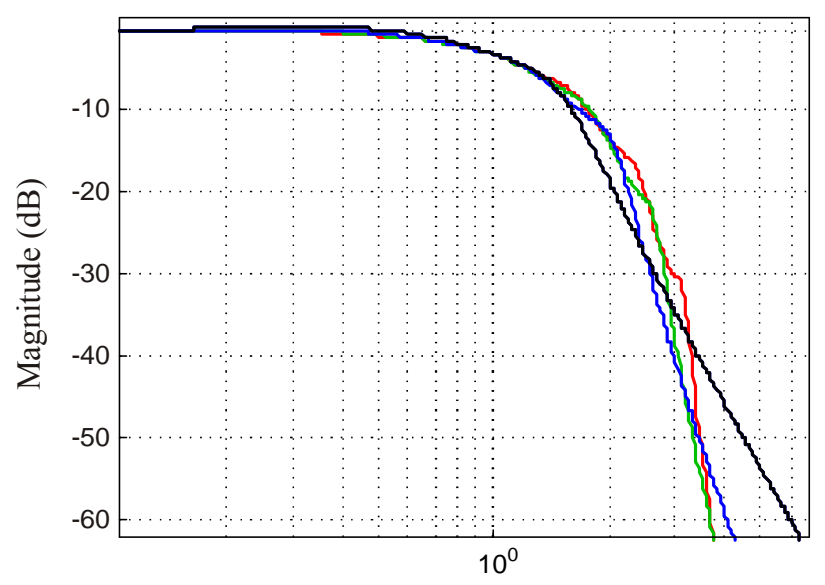

Freqüência $(\mathrm{rad} / \mathrm{s})$

(a)

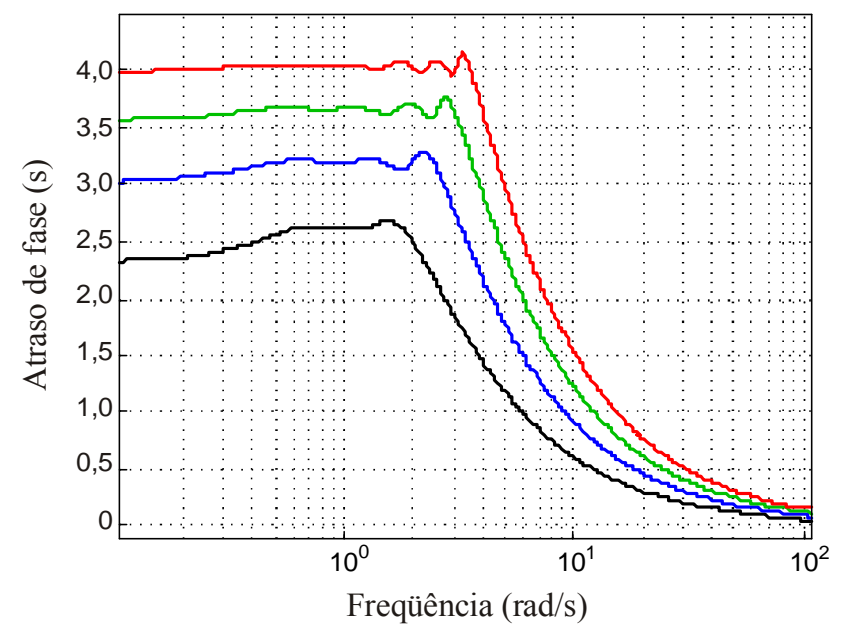

(b)

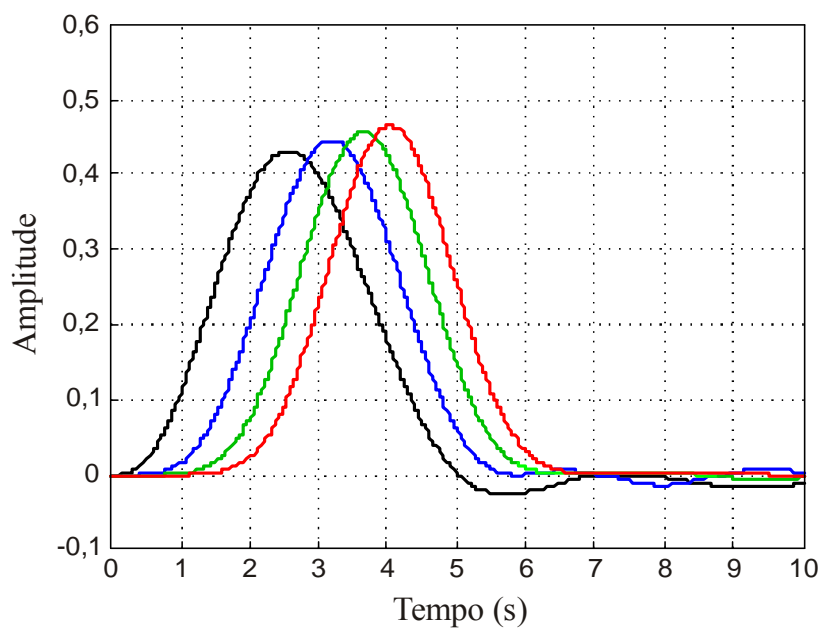

(c)

Fig. 4. Funções FM para $A_{\max }=3 \mathrm{~dB}$ em $1 \mathrm{rad} / \mathrm{s}$. (a) Magnitude; (b) atraso de fase; (c) resposta ao impulso. $n=4$ (preto), $n=6$ (azul), $n=8$ (verde), $n=10$ (vermelho). 


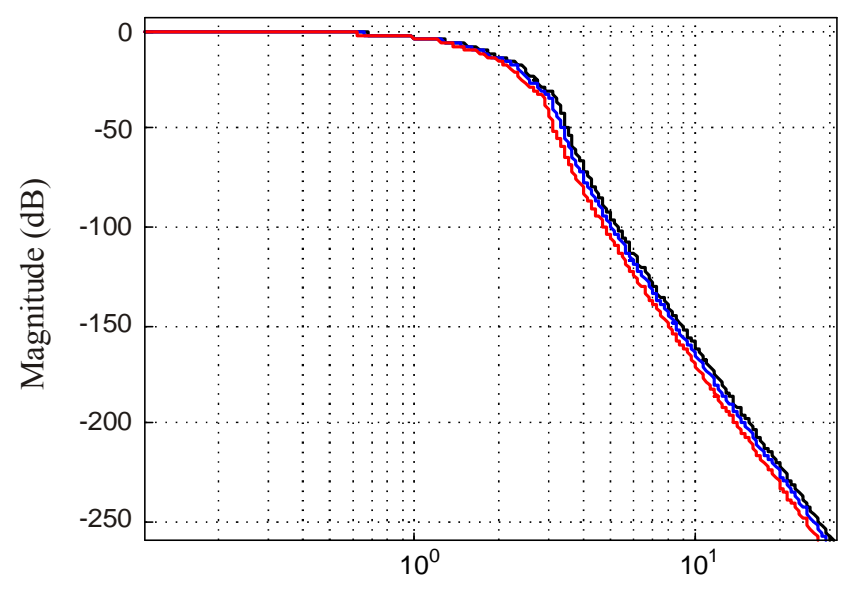

Freqüência $(\mathrm{rad} / \mathrm{s})$

(a)

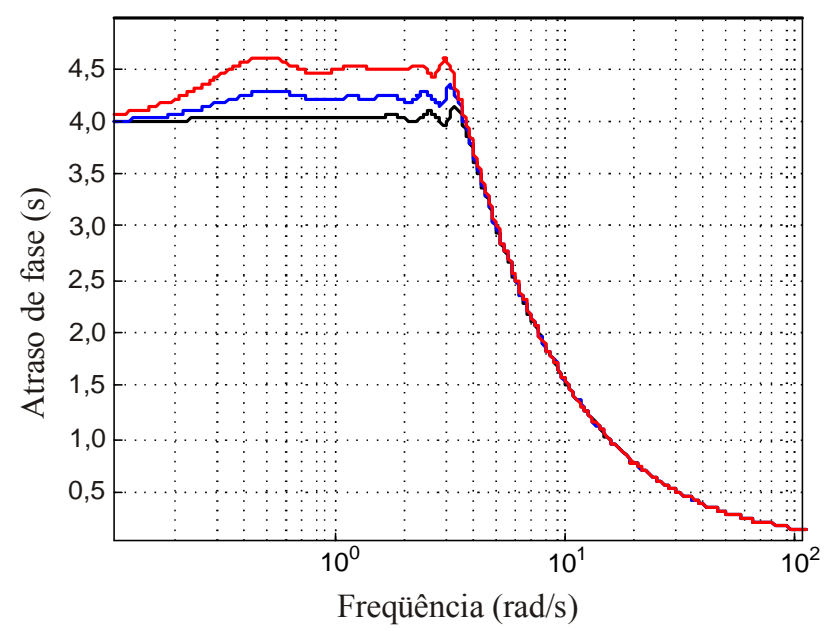

(b)

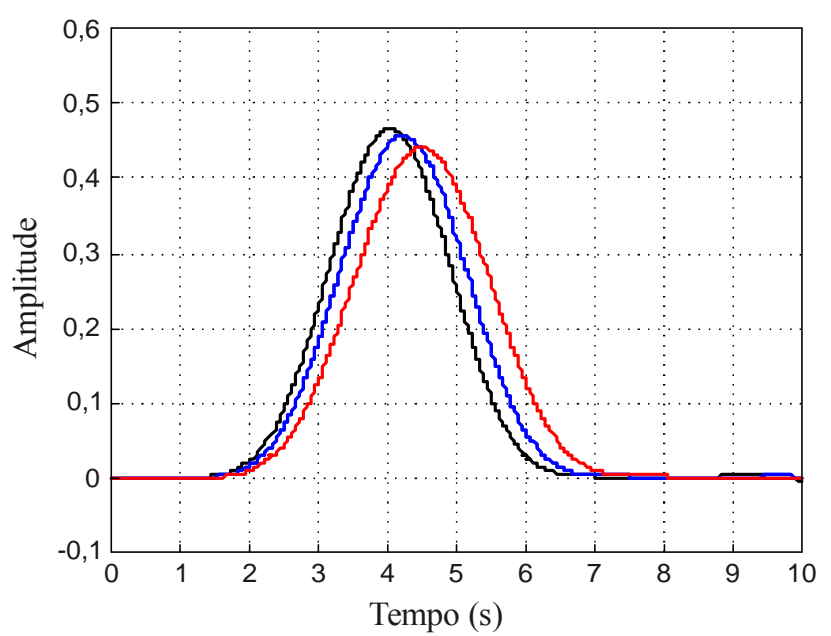

(c)

Fig. 5. Funções FM para $A_{\max }=3 \mathrm{~dB}$ em $1 \mathrm{rad} / \mathrm{s}$. (a) Magnitude; (b) atraso de fase; (c) resposta ao impulso. Com $\mathrm{A}_{2}$ original (preto), com $0,95 \times \mathrm{A}_{2}$ (azul) e $0,9 \times \mathrm{A}_{2}$ (vermelho).

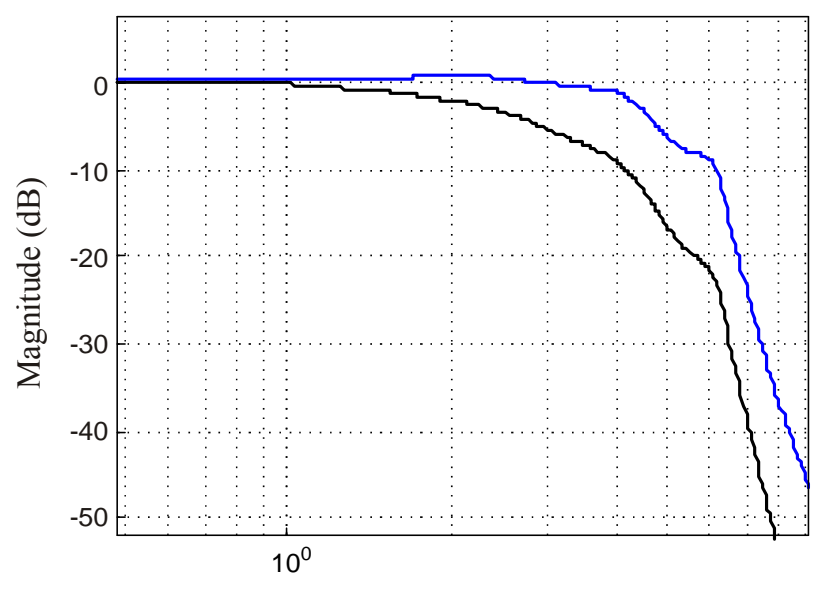

Freqüência $(\mathrm{rad} / \mathrm{s})$

Fig. 6. Exemplo do aumento da banda sem alteração da fase. Função original (preto), função com zeros reais (azul).

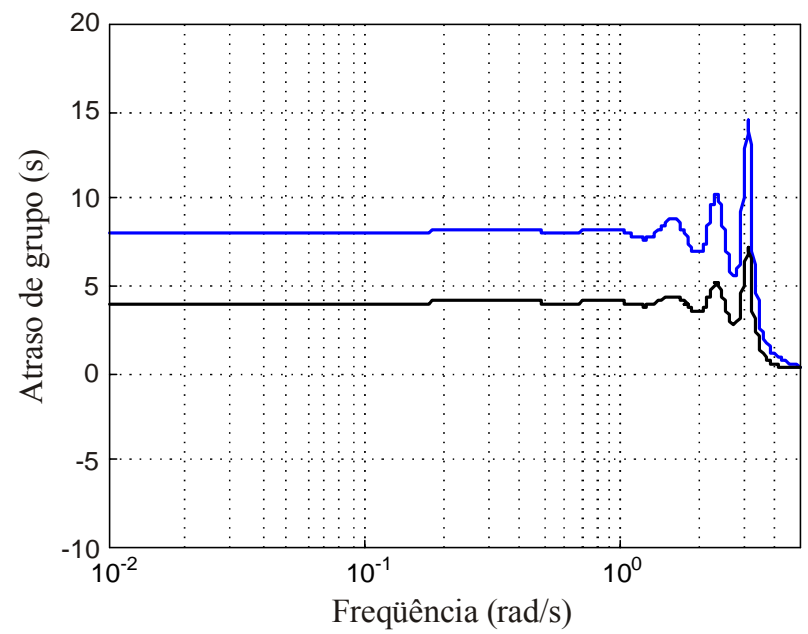

Fig. 7. Exemplo do aumento da banda dobrando o valor do atraso. Função original (preto), passa-tudo gerado a partir da função original (azul).

\section{REFERÊNCIAS}

[1] L. D. Philipp, A. Mahmood, and B. L. Philipp, “An improved refinable rational approximation to the ideal time delay," IEEE Trans. Circuits and Systems, vol. 46, n5, pp. 637-640, May 1999.

[2] I. M. Filanovsky and P. N. Matkhanov, "Synthesis of time delay networks approximating the pulse response described by an integer power of a sinusoid over its semi-period," Analog Integrated Circuits and Signal Processing, vol. 28, no. 1, pp. 83-90, Jul. 2001.

[3] S. M. Kashmiri, S. A. P. Haddad, and W. A. Serdijn, "Highperformance analog delays: surpassing Bessel-Thomson by Pade-approximated Gaussians," in Proc. IEEE Int. Simp. Circuits and Systems (ISCAS), Island of Kos, Greece, May 2006, pp. 2349-2352.

[4] W. E. Thomson, "Delay networks having maximally flat frequency characteristics,” Proc. IEEE, vol. 96, pp. 487-490, Nov. 1949. 